Methods The UK Clinical Practice Research Data-link (CPRD) was used to identify 1605 incident cases of SLE from 1997 to 2005 and matched $1: 4$ to 6284 controls by birth year, gender, general practice and year of first continuous registration. Odds ratios (ORs) of comorbidities at diagnosis and hazards ratios (HRs) after diagnosis of SLEs were estimated adjusting for age, sex, diagnosis year, body mass index, smoking and alcohol consumption.

Results SLE was associated with a higher risk for pre-existing comorbidities, with adjusted ORs $(95 \%$ confidence interval [CI]) of 2.25 (1.97-2.56), $3.37(2.49-4.57)$ and 3.54 (1.896.63 ) for the Charlson index of $1-2,3-4$ and $\geq 5$, respectively. SLE was associated with an adjusted HR (95\% CI) of 1.30 (95\% CI, 1.13-1.49) for developing new comorbidity after the SLE diagnosis. SLE was associated with a greater risk for cancer, cardiovascular, renal, liver, rheumatological and neurological diseases as well as hypothyroidism, psychosis and anaemia. The development of comorbidities was most frequent in the first two years of SLE diagnosis. Patients with SLE also had high risk of death compared with the control group, with a HR of 1.91 (95\% CI, 1.62-2.26).

Conclusions SLE patients had a burden of pre-existing comorbidities at diagnosis and the risk of development of multiple comorbidities were higher after the diagnosis compared to matched controls.

\section{THE PHARMACOTHERAPEUTIC PATTERN OF LUPUS NEPHRITIS PATIENTS AND ITS EFFECT ON THE HOSPITALISATION COST IN CHINA}

T. Li. Renji Hospital- Shanghai JiaoTong University School of Medicine, Department of Rheumatology, Shanghai, China

\subsection{6/lupus-2017-000215.172}

Background and aims To investigate the pharmacotherapeutic pattern of patients with lupus nephritis in China and its impact on the hospitalisation cost.

Methods Data were identified by the primary diagnosis of lupus nephritis from the electronic medical record system for retrospective analysis. All treatment drugs were divided into rheumatic drugs and non-rheumatic drugs for study. The hospitalisation expenses, drug utilisation rate, and its impact on the total cost were analysed.

Results 305 patients with LN between January 2014 and December 2015 were included in this study. The average hospitalisation cost was $\$ 2109.26$, including medical service fees, nursing fees, diagnosis fees, etc. Among them, drug cost was accounted for the high proportion (\$1041.41, 49.37\%), of which the non-rheumatic drugs were accounted for the major proportion (\$892.16,87.70\%). In non-rheumatic drugs, alimentary system drug had the high utilisation rate $(\$ 147.74$, 95.03\%); antimicrobial drugs had the most per person cost $(\$ 581.76,55.30 \%)$. According to the result of principal component analysis, the first principal component, which contains antimicrobial drugs and the alimentary system drugs, accounted for $77.3 \%$ cumulative variance contribution rate. Therefore, the cost of the first principal component had a great impact on the total cost. Based on result of the association analysis, the prescription of $\$ 1$ GCs would produce $\$ 2.76$ alimentary system drugs and $\$ 2.76$ anti-osteoporosis drugs to prevent adverse reactions of GCs.
Conclusions Decrease in the cost of antibiotics and alimentary system drugs could significantly reduce the patient's hospitalisation expenses. To save the disease burden, the cost of drugs for the prevention of GC adverse reactions also should be properly managed.

\section{ELDERLY SLE PATIENTS WITH PREVIOUS SKIN HERPES INFECTION AND HIGHER DOSE OF STEROID ENHANCED THE RISK OF SEVERE HERPES SIMPLEX INFECTION: A NATIONWIDE STUDY}

${ }^{1,2} \mathrm{TH}$ Li*, ${ }^{3}$ YS Chang, ${ }^{4} \mathrm{CC}$ Lai, ${ }^{4} \mathrm{CY}$ Tsai. ${ }^{1}$ Taichung Venterans general hospital - Chiayi branch, Division of Allergy- Immunology- and Rheumatology- Department of Medicine, Chiayi City, Taiwan R.O.C; ${ }^{2}$ National Yang-Ming University, Institute of Clinical Medicine, Taipei, Taiwan R.O.C; ${ }^{3}$ Shuang-Ho Hospital- Taipei Medical University, Division of AllergyImmunology- and Rheumatology-Department of Internal Medicine, New Taipei City, Taiwan R.O.C; ${ }^{4}$ Taipei Veterans General Hospital, Division of Allergy- Immunology- and Rheumatology-Department of Medicine, Taipei City, Taiwan R.O.C

\subsection{6/lupus-2017-000215.173}

Background and aims Systemic lupus erythematosus (SLE) patients are susceptible to herpes simplex virus (HSV) infection, which is occasional but often leading to overwhelming disease such as encephalitis and keratitis. However, only few attempts have been made at the associated incidence and risk factors.

Methods We enrolled SLE patients from the Taiwanese National Health Insurance research database between 1997 and 2012. We compared the incidence rate (IR) of severe HSV infection, including viral septicemia, meningoencephalitis, ocular infection, visceral infection and those with complications after infection, with those of non-SLE cohort. We also evaluated the risk factors of severe HSV infection by means of Cox multivariable proportional hazards model.

Results A total of 122520 subjects (24 504 SLE patients and 98016 age- and gender-matched subjects as control group) were analysed and revealed a significantly higher IR of severe HSV infection in SLE (Incidence rate ratio=3.52, $\mathrm{p}<0.001$ ). Previous skin infection $(\mathrm{HR}=2.17, \mathrm{p}=0.047)$, intravenous steroid pulse therapy $(\mathrm{HR}=4.48, \mathrm{p}<0.001)$, oral daily steroid dose over $7.5 \mathrm{mg}$ prednisolone or equivalent $(\mathrm{HR}=1.60$, $\mathrm{p}=0.010$ ) were independent risk factors for severe HSV infection in SLE patients, while age $\leq 18(\mathrm{HR}=0.47, \mathrm{p}=0.021)$ was a protective factor.

Conclusions A higher risk of severe HSV infection was observed in SLE patients The risk factors for severe HSV infection were age over 18, previous skin infection, intravenous steroid pulse therapy and an oral daily steroid dose over $7.5 \mathrm{mg}$.

\section{THE RELEVANCE OF VASCULAR EVALUATION IN SYSTEMIC LUPUS ERYTHEMATOSUS. RESULTS FROM AN INCEPTION COHORT}

${ }^{1} \mathrm{R}$ Lizola*${ }^{1} \mathrm{CA}$ Hinojosa, ${ }^{1} \mathrm{H}$ Laparra, ${ }^{1} \mathrm{JE}$ Anaya-Ayala, ${ }^{2} \mathrm{LA}$ Mendoza, ${ }^{2} \mathrm{M}$ Ocampo, 2J Romero-Díaz. "Instituto Nacional de Ciencias Médicas y Nutrición "Salvador Zubirán", Vascular Surgery, Mexico city, Mexico; ${ }^{2}$ Instituto Nacional de Ciencias Médicas y Nutrición "Salvador Zubirán", Rheumatology, Mexico City, Mexico

10.1136/lupus-2017-000215.174

Background and aims Systemic lupus erythematosus (SLE), as a chronic inflammatory disease, has been associated with 
accelerated atherosclerosis. However, studies on the prevalence and impact on the peripheral vascular beds remain scarce.

The aim of this study was to determine the prevalence of vascular affection in SLE patients, defined as an altered anklebrachial index (ABI), carotid intima-media thickness (IMT), doppler-duplex interrogation and clinical evaluation by a vascular surgeon, as well as its relationship to clinical and serological variables.

Methods Eighty consecutive SLE patients ( $\geq 4$ ACR criteria) from an inception cohort were studied. Each patient had a vascular clinical evaluation and was screened for ankle-brachial index (ABI) and carotid intima-media thickness (IMT) after 12.1 (3.8) yrs of follow-up. In patients with a history of deep vein thrombosis (DVT), a doppler-duplex ultrasound interrogation was added to the evaluation.

Results The mean age of the patients was 39 years (SD 9). Of the 80 patients studied, $4 \%$ had an abnormal ABI, $8.7 \%$ had a history of DVT, and $15 \%$ had an altered carotid IMT. The global prevalence of any-kind vascular disease was $21.2 \%$. Variables strongly correlated to vascular disease were current age, age at diagnosis and $\operatorname{IgM}$ anticardiolipin (aCL $\operatorname{IgM}$ ) antibody. Conclusions The increased prevalence of a diverse range of vascular diseases in this young population of patients with SLE justifies a vascular evaluation in every patient to identify, follow and potentially treat them in a timely fashion.

\section{D-DIMER AS AN EARLY MARKER IN PATIENTS WITH LUPUS MESENTERIC VASCULITIS}

X Ma*, X Feng, H Wang, B Hua, L Sun. Drum Tower Hospital - Medical School of Nanjing University, Rheumatology, Nanjing, China

\subsection{6/lupus-2017-000215.175}

Background and aims No early serum marker contributes to the diagnosis of lupus mesenteric vasculitis (LMV). The aim of this study was to investigate clinical significance of serum d-dimer level as an early diagnosis marker of LMV patients.

Methods The 57 systemic lupus erythematosus (SLE) patients were retrospectively analysed and classified into LMV group $(n=19)$ and Non-LMV group $(n=38)$ between May 2010 and January 2016. The serum d-dimer level was measured on the first day after SLE patients presented acute abdomen as well as imaging, other laboratory-testing parameters, and SLEDAI during the same period. The maximum and mean d-dimer values were analysed and compared with other potential markers for diagnosis of LMV. The correlation of d-dimer level with other potential severity markers and inflammation parameters were also studied.

Results Both maximum and mean d-dimer level on the first day of presentation of acute abdomen were significantly higher in LMV patients. The d-dimer level was correlated well with 1-lactate and SLEDAI. In addition, d-dimer level was detected poor correlation with white blood cell count and Creactive protein level.

Conclusions d-dimer level could be an effective and early serum diagnosis marker of LMV.

\section{ACCRUAL OF DISEASE COMORBIDITIES OVER 8 YEARS IN A MULTICENTRE INCEPTION SLE COHORT}

${ }^{1} \mathrm{M}$ Urowitz, ${ }^{1} \mathrm{D}$ Gladman*, ${ }^{2} \mathrm{~J} \mathrm{Su},{ }^{2} \mathrm{~N}$ Anderson. Systemic Lupus International Collaborating Clnics; 'University of Toronto, Medicine, Toronto, Canada; ${ }^{2}$ Toronto Western Hospital, Rheumatology, Toronto, Canada

\subsection{6/lupus-2017-000215.176}

Background and aims The annual accrual of comorbidities in patients with SLE is not well described. We report the annual occurrence of these features in an inception cohort of patients with SLE.

Methods An international research network comprised of 33 centres from 11 countries has followed an inception cohort of SLE patients 2yearly according to a standardised protocol between 2000 and 2016. Of these, 717 patients followed for a minimum of 8 years constitute the study population. Comorbidities including atherosclerotic vascular events (AVEs), osteoporosis, osteonecrosis and diabetes are assessed using the SLICC/ACR Damage Index (SLICC/DI). AVEs are described and attributed on a specialised form. Diagnosis of an event is confirmed using standard clinical criteria, relevant laboratory data and imaging where appropriate. Descriptive statistics were used.

Abstract 176 Table 1 Cumulative SDI, AVEs, steoporosis, osteonecrosis and diabetes in the firest 8 years of follow-up ( $n=17)$.

\begin{tabular}{|l|c|c|c|c|c|}
\hline $\begin{array}{c}\text { Follow- } \\
\text { up }\end{array}$ & $\begin{array}{c}\text { SDI } \\
\text { (meantstd) }\end{array}$ & AVE & Osteoporosis & Osteonecrosis & Diabetes \\
\hline 1 & $0.3 \pm 0.7$ & $4(0.5 \%)$ & $3(0.4)$ & $3(0.4 \%)$ & $13(1.8 \%)$ \\
\hline 2 & $0.5 \pm 1.0$ & $5(0.7 \%)$ & $5(0.7 \%)$ & $10(1.4 \%)$ & $17(2.4 \%)$ \\
\hline 3 & $0.6 \pm 1.2$ & $10(1.4 \%)$ & $7(1.0 \%)$ & $15(2.1 \%)$ & $17(2.4 \%)$ \\
\hline 4 & $0.7 \pm 1.2$ & $13(1.8 \%)$ & $10(1.4 \%)$ & $18(2.5 \%)$ & $19(2.7 \%)$ \\
\hline 5 & $0.8 \pm 1.3$ & $18(2.5 \%)$ & $11(1.5 \%)$ & $21(2.9 \%)$ & $19(2.7 \%)$ \\
\hline 6 & $1.0 \pm 1.4$ & $20(2.8 \%)$ & $15(2.1 \%)$ & $25(3.5 \%)$ & $21(2.9 \%)$ \\
\hline 7 & $1.0 \pm 1.4$ & $21(2.9 \%)$ & $17(2.4 \%)$ & $27(3.8 \%)$ & $22(3.1 \%)$ \\
\hline 8 & $1.1 \pm 1.5$ & $25(3.5 \%)$ & $19(2.7 \%)$ & $31(4.3 \%)$ & $25(3.5 \%)$ \\
\hline
\end{tabular}

